\title{
Aplicación del modelo de Nola Pender a un adolescente con sedentarismo
}

\author{
Fernando Trejo Martínez.
}

RESUMEN

Se estudio el caso de un adolescente con sedentarismo y malos hábitos dietéticos, operacionalizando los conceptos principales del Modelo de Promoción a la Salud de la doctora Nola Pender, se construyo un instrumento con base a los conceptos del modelo y se aplicó al adolescente para identificar los motivos por los cuáles un joven no hacía ejercicio y consumía alimentos chatarra, se realizó intervención de enfermería enfocada a la promoción a la salud, en especial en los beneficios del ejercicio y una dieta balanceada, se dio seguimiento a los seis meses y al año, obteniéndose como resultado, que el adolescente modificara sus hábitos alimenticios, empezar a hacer ejercicio con lo que ha disminuido su índice se masa corporal (IMC) de 27.4 a 23.5 y su peso de 84 a 72 kg ubicándose dentro de su peso ideal para su edad.

Palabras clave: modelo de promoción a la salud, conducta saludable, Nola Pender, adolescente.

\section{Aplication of health promotion model from Nola Pender in a sedentary teenager}

\begin{abstract}
Health case study, for a teenager with sedentary and dietetic wrong habits, putting in practice the main concepts of Health Promotion Model from Dr. Nola Pender. A device with model's concepts was built and applied to the teenager to find out reasons why a young guy was not doing exercise and he was eating low nutrition food. Nurses were active focusing in the promotion of health, especially in letting the patient know about benefits regarding exercise and balanced diet. Follow up was given within intervals of 6 months and 1 year. As a result the teenager had modified his alimentary habits, started to make more exercise, consequently his CMI (corporal mass index) decreased from 27.4 to 23.5 and he had a weight decrease from 84 to $72 \mathrm{~kg}$, positioning him within the ideal percentile of his age.
\end{abstract}

Key words: health promotion model, healthy habits, Nola Pender, teenager.

Correspondencia: Fernando Trejo Martínez. Instituto Nacional de Neurología y Neurocirugía. Laboratorio de Investigación Sociomédica. Av. Insurgentes Sur \# 3877 Col. La Fama. 14269. México, D.F. E-mail: fertre001@yahoo.com.mx

$\mathrm{E}$ 1 estilo de vida condiciona gran parte de la morbilidad en muchos países según Cabezas-Peña ${ }^{1}$, durante siglos el estilo de vida del ser humano ha estado dotado de un componente de actividad física, al tener que desarrollar trabajos y labores para su subsistencia ${ }^{1}$; sin embargo, en la actualidad el aumento de la tecnología facilita las tareas cotidianas, donde no es necesario realizar tareas físicas extenuantes para satisfacer las necesidades vitales, lo que ocasiona que el ser humano al habitar en grandes urbes; se vuelva sedentario y por lo tanto, es un gran riesgo para innumerables enfermedades.

Las principales enfermedades son de origen cardiovascular, como la hipertensión arterial que condiciona infarto al miocardio y cerebral, y si se le agrega otro factor condicionante como una alimentación rica en grasas, con lleva a sobrepeso, esto se vuelve una bomba de tiempo para la salud de los individuos con estos factores. 
En México la causa número uno de morbi-mortalidad son las enfermedades del corazón ${ }^{2}$ con 111,025 casos en el $2005^{3}$ siendo la tercera causa de morbilidad hospitalaria en el mismo año ${ }^{4}$ además de que cada día la tasa va en aumento, el problema del sedentarismo se vuelve una amenaza para la salud de los individuos con ingesta de alimentos chatarra, característica de los adolescentes de hoy, esto puede explicar las estadísticas antes mencionadas. Otro factor que tal vez no es tomado en cuenta es el estrés provocado por conflictos de personalidad propios de la edad.

Son muchos los factores que condicionan lo antes planteado, por esto es que el Modelo de Promoción a la Salud de la doctora Nola Pender ${ }^{5}$, nos ofrece la posibilidad de evaluar a la persona que tiene riesgo de sufrir una desviación de la salud producida por la suma de éstos factores, y así poder actuar antes de que se produzcan alteraciones que lleven a las personas a una atención hospitalaria, con riesgo para la vida y función.

La aplicación del Modelo de Pender, ofrece la posibilidad de llevar a cabo una adecuada promoción a la salud antes de que se presenten complicaciones que pongan en riesgo a la población, el presente estudio es un intento de operacionalizar el modelo y hacerlo funcional para los profesionales de enfermería sobre todo a los que tienen como tarea la atención primaria de la comunidad.

\section{Modelo de la Nola Pender}

El modelo de promoción a la salud de Pender, esta basado en la concepción de la promoción de la salud, que es definida como aquellas actividades realizadas para el desarrollo de recursos que mantengan o intensifiquen el bienestar de la persona.

El modelo surge como una propuesta de integrar la enfermería a la ciencia del comportamiento, identificando los factores que influencian los comportamientos saludables, además de una guía para explorar el complejo proceso biopsicosocial, que motiva a los individuos para que se comprometan en comportamientos promotores de la salud $\mathrm{d}^{6}$.

El modelo de la promoción a la salud es básicamente un modelo de enfermería, para evaluar la acción de promoción a la salud y el comportamiento que conduce a la promoción a la salud y de la interrelación entre tres puntos principales:

1. Características y experiencias de los individuos.

2. Sensaciones y conocimiento en el comportamiento que se desea alcanzar.

3. Comportamiento de la promoción de la salud deseable ${ }^{9}$.

En la construcción del modelo el autor uso como bases teóricas, la teoría de aprender social de Montgomery y modelo para evaluar expectativas, ambas teorías derivadas de la psicología.

\section{Principales conceptos del modelo}

Esta dividido en tres dimensiones con factores que condicionan la promoción a la salud una cada dimensión.

1. Conducta previa relacionada: la frecuencia de la misma conducta o similar en el pasado, efectos directos o indirectos de la probabilidad de comportarse con las conductas de promoción a la salud.

2. Factores personales: se refiere a todos los factores relacionados con las personas que influyen en el individuo para que se relacione con su medio para desarrollar conductas promotoras de salud que incluyen factores biológicos, psicológicos y socioculturales, además de los beneficios percibidos de las acciones promotoras de salud, así como las barreras que encuentra para estas conductas.

3. Influencias situacionales: son las percepciones y cogniciones de cualquier situación o contexto determinado que pueden facilitar o impedir la conducta.

4. Factores cognitivo-preceptúales: son mecanismos motivacionales primarios de las actividades relacionadas con la promoción de la salud.

\section{Presentación del caso}

Se trata de un adolescente sexo masculino de 17 años de edad, hijo primogénito, el padre y la madre trabajadores ambos, con estudios de licenciatura, habitan en vivienda propia con trabajo estable y una clasificación que entra dentro de medio socioeconómico medio-medio.

Estudiante de preparatoria en su $2^{\circ}$ año, tiene el turno vespertino, por lo que las mañanas, se queda sólo ya que sus padres trabajan y su hermana estudia en horario matutino.

Su rutina consiste en levantarse tarde, aproximadamente hasta las 10 ó 11 de la mañana, desayuna o almuerza sólo, realiza actividades escolares y en ocasiones quehaceres de la casa (arregla su habitación, va por víveres, etc.) prepara sus útiles escolares, se baña y se va a la escuela, cumple con sus actividades académicas, en su ambiente escolar, algunas veces no entra a clases y pasea con los amigos fuera de la escuela, y consume alimentos ricos en carbohidratos y grasas, (tortas, tacos, hamburguesas, y frituras, además de refrescos embotellados), regresa a su domicilio alrededor de las $22 \mathrm{hs}$ cena junto con sus padres aunque ellos no consumen alimentos, sólo lo acompañan, en ocasiones realiza tareas escolares pero la mayoría de las veces ve televisión en su cuarto hasta altas horas de la noche 1 ó $2 \mathrm{am}$. 
La alimentación de la familia es balanceada y cuando sus actividades lo permiten comen en juntos.

Actualmente no practica ningún deporte ni actividad física, aunque sus padres le insisten que lo haga, hasta hace un año realizaba actividad física importante, con sus padres, practicaba frontenis y realizaba joging de tres a cuatro veces por semana, con lo que se mantenía un peso adecuado.

\section{MATERIAL Y MÉTODOS}

Sampieri define, el estudio de caso como una investigación sobre un individuo, grupo, organización, comunidad o población, que es visto y analizado como una entidad, se menciona que es una investigación mediante procesos cuantitativo, cualitativo o mixto, se analiza profundamente una unidad para responder el planteamiento del problema, probar una hipótesis y desarrollar teoría ${ }^{8}$.

En este sentido se analiza un individuo con el enfoque del modelo de promoción a la salud de Nola Pender.

Se construyó un instrumento de recolección de datos, con base en los conceptos principales del modelo para determinar porque el adolescente no realiza conductas de promoción a la salud.

Se utilizó metodología como menciona Fawcett ${ }^{9}$, se fue de lo complejo a lo concreto, para el diseño de un instrumento de valoración de enfermería, enfocado en detectar riesgos de desviación de la salud, formular diagnósticos de enfermería, reales y de riesgo para elaborar un plan de intervención. Así mismo se realizo la operacionalización de los conceptos como lo mencionan Waltz, Strickland y Lenz ${ }^{10}$, para adquirir indicadores empíricos de los conceptos del modelo y de esta manera obtener los ítems, para la construcción del instrumento de valoración.

El instrumento consta de 42 ítems, divididos en dos secciones y de 21 ítems cada una, elaborada en sentido positivo y la segunda en sentido negativo, todos los ítems tienen 4 opciones para contestarse, las respuestas son tipo Likert y van desde nunca, esporádicamente, casi nunca y nunca se les dio el valor del 1 al 4 donde nunca es 1 , esporádicamente es 2 , casi nunca es 3 y siempre es 4 .

Se puede calificar en forma general, haciendo una operación algebraica donde se suman todos los valores del instrumento, el valor máximo de calificación es de 105, sí se suman todos los valores de la sección positiva que es de 84 y con los valores de la sección negativa el valor máximo es 21 , entonces si la persona tiene una calificación máxima de 105 quiere decir que tiene conductas promotoras de la salud, pero si al hacer la suma se obtiene un resultado de 91 a este resultado se le resta al valor ideal (105) el resultado será de $14(105-91$ = 14).

Para determinar si la persona es merecedora de intervención de promoción a la salud por parte del profesional de enfermería, se establecen los siguientes rangos.

1. No intervención sólo reforzamiento de conductas promotoras de 105 a 100.

2. Intervención moderada de 99 a 90 .

3. Intervención exhaustiva de 89 a 80 .

4. intervención urgente de 79 y menos de 79 .

Además el instrumento cuenta con otras dos secciones una A y otra B.

La sección A: se refiere a datos sociodemográficos incluyendo signos vitales, peso, talla e índice de masa corporal ${ }^{11-13}$.

La sección B: esta dedicada a la exploración física, conteniendo los cuatro puntos principales de la exploración, observación, palpación, percusión y auscultación.

Todas las secciones del instrumento nos servirán para relacionar los datos y así obtener un panorama general del paciente.

El instrumento y valoración se aplicaron en tres momentos al inicio del estudio, a los tres meses y al año. Después de cada valoración se realizaron diagnósticos de enfermería y un plan de intervenciones de acuerdo a los diagnósticos.

Se realizo consultoría en enfermería ${ }^{14}$ visitando al adolescente durante dos veces a la semana por dos semanas para la aplicación del instrumento y exploración física. Se realizó consultoría cada mes hasta cumplir el año.

\section{RESULTADOS}

Los resultados obtenidos fueron en tres momentos el primer momento se realizo al inicio del estudio. La aplicación del instrumento arrojo la calificación de 90, lo que indica que necesitaba intervención moderada, además se complemento la información con la medición del peso corporal $(81 \mathrm{~kg})$, talla $(1.75 \mathrm{~m})$ del adolescente y en con base en estos datos se calculo el índice de masa corporal (IMC) que fue de 26.5 que de acuerdo a la OMS, el adolescente esta en una etapa de preobeso.

Estos resultados se complementaron con la sección B del instrumento referida a la exploración física, y lo sobresaliente en la observación arrojo los siguientes datos, el adolescente es de complexión fornida, pero con un abdomen poco globoso a expensas de panículo adiposo, rostro regordete, el resto de la exploración resulto normal. 
Se obtuvieron dos diagnósticos de enfermería

1. Riesgo de obesidad, relacionada con ingesta de alimentos ricos en grasas.

2. Riesgo de obesidad, relacionada con falta de ejercicio.

\section{Plan de cuidados}

Diagnóstico de enfermería: riesgo de obesidad, relacionada con ingesta de alimentos ricos en grasas.

\section{Intervenciones de enfermería}

Diseñar un plan de acción para mejorar la autoeficacia percibida*, respecto a adoptar un compromiso con un plan de acción* enfocado en un cambio de hábitos alimenticios.

Nota* los elementos marcados con asterisco, son conceptos teóricos del modelo de Promoción a la Salud de Nola Pender.

\section{Acciones de enfermería}

- Medición de peso, talla e índice de masa corporal.

- Elaboración de un plan dietético de reducción de peso.

- Sugerir ingesta de 5 comidas al día (desayuno, comida y cena, además de 2 colaciones entre comidas).

- Evitar ingesta de alimentos preparados fuera del hogar.

- Estimular ingesta de frutas o verduras entre comidas si tiene apetito.

- Sugerir ingesta de por lo menos dos litros de agua al día.

- Concienciar al adolescente para que sea un agente de cambio entre su grupo de amigos y evitar el consumo de alimentos chatarra.

- Sugerir que el adolescente lleve alimentos preparados en casa.

\section{Evaluación}

El adolescente bajo tres kilos de peso el primer mes, y uno el segundo, pero para el tercero había recuperado dos kilos, lo que en total en tres meses sólo bajo dos kilos, menciona que le fue muy difícil mantener la dieta, porque sus amigos lo incitaban a comer alimentos ricos en grasa y carbohidratos, además de que sus padres continuaban comiendo, como lo hacían antes y que también le resultaba difícil realizar dieta cuando la familia come algo diferente a él.

Diagnóstico de enfermería: riesgo de obesidad, relacionada con falta de ejercicio.

Intervenciones de enfermería: diseñar un plan de acción para mejorar la autoeficacia percibida, respecto a adoptar un compromiso con un plan de acción, enfocado a estimular la realización de ejercicio físico constante.

\section{Acciones de enfermería}

- Educación sobre beneficios del ejercicio.

- Estimular al adolescente para que forme parte de un equipo deportivo y lo practique constantemente.

- Informarle de los beneficios de la caminata en los parques.

- Realizar un plan de actividades físicas que se pueda realizar en el hogar.

\section{EVALUACIÓN}

El adolescente inicio el primer mes realizando caminatas en el parque junto con su hermana, pero sólo los días que no acudían a clases, refiere que intento participar en un equipo de fútbol, pero no le gusta y no le entiende lo dejo a las dos semanas, manifiesta además que no le gusta hacer ejercicio sólo y que su padres no lo pueden acompañar, primero por la incompatibilidad de horarios y después porque su padre esta enfermo y le restringieron actividades físicas por un tiempo.

Evaluación general: a pesar que el adolescente fue inconstante con el plan dietético y con el programa de ejercicios, bajo dos kilos lo que lo saco de la etapa de preobeso (OMS); sin embargo, existen factores importantes de acuerdo al modelo de Nola Pender como: barreras percibidas de acción (son bloqueos anticipados, imaginarios o reales, y costes personales de la adopción de una conducta determinada) barreras percibidas para las conductas promotoras de salud. La creencia del individuo de que una actividad o conducta es difícil o inviable que puede influir en su intención de adoptar una conducta saludable. Por lo tanto se tuvo que rediseñar el plan de intervenciones para lograr que el adolescente adquiriera acciones promotoras de salud.

\section{Segundo momento (a los tres meses)}

La aplicación del cuestionario arrojo una calificación de 93, el peso se redujo a $79 \mathrm{~kg}$ y el índice (IMC) fue de 25.8, la exploración física casi no vario en los rubros de observación y palpación. De acuerdo a estos resultados el adolescente se encontraba en la etapa de sobrepeso (OMS) y de acuerdo al instrumento, aún necesitaba intervención moderada.

Debido a que el plan de intervenciones y acciones de enfermería, no resultaron positivas, se rediseño el plan de intervenciones, en este caso se incluyo a toda la familia, en el 
diagnóstico de enfermería y se realizo un plan familiar.

\section{Diagnóstico de enfermería}

1. Riesgo de sobrepeso relacionado con la ingesta de alimentos ricos en grasas.

2. Riesgo de sobrepeso, relacionado con la falta de ejercicio.

Intervenciones de enfermería. diseñar un plan de acción enfocado a la familia para lograr que el adolescente adquiera conductas promotoras de salud, referentes a la ingesta de una alimentación sana.

\section{Acciones de enfermería}

- Se diseño un régimen dietético que incluía ingesta de verduras y frutas en los tres alimentos de la familia.

- Se les informo de la importancia de realizar cinco comidas al día, (desayuno, comida, cena y dos colaciones entre comidas).

- Se les educo acerca del riesgo de la ingesta excesiva de grasas y comidas ricas en carbohidratos.

- Se les comento que es importante la convivencia familiar durante los alimentos.

- Se incluyo la ingesta de por lo menos dos litros de agua al día.

Diagnóstico de enfermería: riesgo de sobrepeso, relacionado con la falta de ejercicio.

Intervenciones de enfermería: realizar un programa de ejercicios familiar que incluya caminatas por los parques y adaptar el hogar para poder tener una actividad física en el.

\section{Acciones de enfermería}

- Se elaboró un calendario que incluía los momentos en que algún miembro de la familia pudiera acompañar al adolescente para que juntos realizaran caminatas en parques.

- Se realizó otra visita domiciliara para adaptar un espacio en el hogar que pudiera servir para realizar ejercicio cuando el clima o las actividades de la familia no dieran oportunidad de hacerlo fuera de casa.

- Se sugirió que realizarán actividades juntos como jugar en grupo, en el parque o en el hogar.

\section{Tercer momento (al año de iniciado el estudio):}

La aplicación del cuestionario presento ahora una calificación de 100 , el peso se redujo hasta $74 \mathrm{~kg}$ y el IMC fue de 24.16 lo que lo coloca en la etapa de normal según la OMS. De la exploración física se obtuvieron los siguientes datos, a la observación se ve un adolescente con cuerpo atlético, sin abdomen a simple vista, a la palpación se observa aumento de masa corporal en las cuatro extremidades y el abdomen ya no se palpa panículos de grasa.

Ya no se obtienen diagnósticos de enfermería pués se corrigió la preobesidad y sobrepeso, inclusive se nota un adolescente con más seguridad y responde con acertividad y rapidez el cuestionario. La relación familiar se ve más armónica y ahora buscan cualquier momento para realizar actividades físicas juntos, el padre ha empezado a realizar ejercicio y se siente mejor.

Sus hábitos alimenticios han cambiado, ahora todos siguen un régimen alimenticio más sano, han restringido ingesta de alimentos ricos en grasas y carbohidratos, por lo que todos han bajado de peso.

\section{CONCLUSIONES}

El estudio de este caso fue una forma de operacionalizar la teoría de enfermería a la realidad de un adolescente, con base en los conceptos teóricos se pudo analizar cuales eran las actitudes y conductas que influían para no realizar conductas saludables; no obstante, hubo que replantear las intervenciones de enfermería porque a primera instancia el plan no funciono.

El seguimiento a base de la consultoría pudo lograr identificar las barreras que el adolescente presentó para no adoptar el primer plan y así cambiar su estilo de vida, cabe señalar que la persona no es una entidad unitaria, y que esta relacionada siempre con sus semejantes, además las conductas se aprenden en el seno familiar, así se logro identificar las barreras de la relación con la convivencia del adolescente con su familia y compañeros de escuela, por lo tanto se ajusto el plan de intervenciones y las acciones de enfermería estuvieron dedicadas al núcleo familiar.

Esto favoreció que toda la familia apoyara al adolescente para lograr un cambio de estilo de vida y adquirieran conductas promotoras de la salud.

Hoy el adolescente ha logrado eliminar sus barreras para adoptar una conducta saludable y esto influyo en el alza de su autoestima al saber que es capaz de lograr un objetivo y que cuenta con el apoyo de su familia para lograrlo.

Nola Pender, diseño su modelo para evitar que las personas lleguen por una complicación a un hospital, adquiriendo conductas promotoras de la salud, además es un modelo que a diferencia de otros que están elaborados para el cuidado de la persona enferma, y este modelo se enfoca en el manteni- 
miento de la salud, el profesional de enfermería puede utilizarlo para lograr que la población que cuida adopte un estilo de vida que le garantice su salud en lugar de cuidar su enfermedad.

\section{REFERENCIAS}

1. Cabezas-Peña C. Recomendaciones de expertos del PAPPS, sobre el estilo de vida. Atención primaria, $2005 \mathrm{~N}^{\circ} 36$ (suplem. 2).

2. http://www.inegi.gob.mex/est/contenidos/espanol/rutinas/ ept.asp?t=mpob45\&c=3222

3. http://www.inegi.gob.mex/prod_serv/contenidos/espanol/bvinegi/ productos/continuas/vitales/demograficas/2005cua-pob-18pdf

4. 2004 y 2005: SSA. Base de datos sobre egresos hospitalarios 2004 y 2005 por categorías de la CIE 10. Reprocesamiento INEGI.

5. Marriner A, Raile M. Modelos y teorías de enfermería. $6^{\circ}$ Edición, 2006 Ed. Elsevier, España.

6. Jannaina FV. Análise do diagrama de promocao da saúde de Nola J. Pender. Acta Paul Enferm 2005; Vol 18 N³:237.

7. Cid, PH. Factores biológicos y psicosociales del estilo promotor de la salud. Rev. Med. Chile Vol. 34 N$^{\circ} 12$ Santiago dic. 2006; 1492.
8. Hernández-Sampieri R. Metodología de la investigación. $4^{\circ}$ Edición, Editorial McGraw-Hill Interamericana México 2006; p. 224.

9. Fawcwtt J. Analisis an evaluation of contemporary nursing knoweledg. Editorial FA Davis Company Philadelphia. E.U. 2000 p3.

10. Waltz FC. Measurement in Nursing Research, $2^{\circ}$ Edición. Ed. . Davis Company Philadelphia. E. U. 1992. Tomado de la guía de lecturas básicas «modelos y métodos de intervenciones en enfermería» agosto 2006, p 154-98.

11. Comité de Expertos de la OMS sobre el estado físico: el estado físico: uso e interpretación de la antropometría. Serie de informes técnicos, 854. Ginebra (Suiza): OMS, 1995.

12. Comité de Expertos de la OMS sobre la obesidad: obesity: preventing and managing the global epidemic. Report of a WHO consultation on obesity. WHO technical report series, 894. Ginebra (Suiza): OMS, 2000 .

13. Organización Mundial de la Salud: Appropriate body-mass index for Asian populations and itsimplications for policy and intervention strategies. Ginebra (Suiza): Organización Mundial de la Salud, 2004.

14. García-Laborda A. Consultoría de enfermería en salud mental para atención primaria. Cultura de los cuidados. Año V, n. 10 (2. semestre 2001); 30-35. 